\title{
Exploration of User Reactions to Different Dialog-Based Interaction Styles
}

\author{
Birgit Endrass ${ }^{1}$, Christoph Klimmt ${ }^{2}$, Gregor Mehlmann ${ }^{1}$, \\ Elisabeth André ${ }^{1}$, and Christian Roth ${ }^{3}$ \\ 1 Human Centered Multimedia, Augsburg University, \\ Universitätsstr. 6a, D-86159 Augsburg, Germany \\ \{endrass, mehlmann, andre\}@hcm-lab.de \\ http://hcm-lab.de \\ 2 Institut fuer Journalistik und Kommunikationsforschung, \\ Hochschule fuer Musik, Theater und Medien Hannover, \\ Expo Plaza 12, D-30539 Hannover, Germany \\ christoph.klimmt@ijk.hmtm-hannover.de \\ 3 Center for Advanced Media Research Amsterdam, \\ VU University Amsterdam, The Netherlands \\ c.roth79@googlemail.com
}

\begin{abstract}
How human users perceive and interact with interactive story-telling applications has not been widely researched so far. In this paper, we present an experimental approach in which we investigate the impact of different dialog-based interaction styles on human users. To this end, an interactive demonstrator has been evaluated in two different versions: one providing a continuous interaction style where interaction is possible at any time, and another providing system-initiated interaction where the user can only interact at certain prompts.
\end{abstract}

\section{Research Problem}

Interactive stories are envisioned and designed to facilitate positive, enjoyable, and moving experiences in their users [1]. The simple fact that interactive stories rely on user interaction suggests that involving users in the design process is essential for building successful systems with high end-user acceptance. To the extent that solid conceptual reasoning is underlying user-centered formative research, user studies can therefore help system creators make better choices when several design options are available [2].

The present paper illustrates the value of systematic, concept-based user research in designing interactive stories by an examination of user reactions to two different ways of managing text-based dialog between user and story characters. The conceivable modes of managing user dialog come with specific advantages and caveats both from a designer's and from a user's perspective. Exploring potential differences in user reactions to manifestations of different design options 
regarding text-based dialog can help system creators make an informed decision about which kind of dialog option to select. For example, if one mode of managing dialog is particularly costly from a designer's perspective, exploring user reactions to an interactive story prototype that employs this mode will allow concluding whether gains in (enjoyable, engaging) user experiences can be expected that justify the effort of a full-scale implementation of the dialog mode in question in the final system (see also [3]).

For this purpose, we present a dialog-based interactive storytelling prototype that was implemented in two different versions: One version enabling continuous dialog interaction to users, whereas the other version offers round-based, systeminitiated dialog possibilities. With it, we want to investigate in how far the user's freedom of choice or the guidance by the system influences the user's perception of the experience. This is a crucial question in interactive storytelling systems, since the user's autonomy has proven to be an important feature that influences the user's experience of the entertainment [4]. However it is not clear to what extent the design of a system needs to be adapted in order to reach a feeling of autonomy.

\section{Dialog-Based Interaction in a Virtual Scenario}

In order to test the impact of different interaction styles on human users, we implemented a storytelling application using virtual characters that is able to cope with a user's typed text input and provides appropriate reactions. Therefore several components were needed, such as language understanding to parse the user's text input into abstract dialog utterances [5], an authoring tool that allows us to model different interaction strategies [6] as well as a graphical representation holding the virtual characters [7].

For our demonstrator, we chose to model a soap-like story, where the characters are involved in a romantic conflict. The user, who is represented by an avatar, can interact with a group of girls, a group of guys, or a waitress that is working in the Virtual Beergarden scenario. Through observation and interaction, the user will learn that there is a love story secretly going on. Dependent on the user's interactions, the characters will reveal their love, ask for help and follow the user's advice. For different outcomes of the game, the constellations of the conversational agent groups can change. In that manner, one of the guys can, for example, walk over to the waitress and ask her out for a date.

To investigate the impact of different dialog-based interaction styles on human users, we implemented two different versions of the application that are described in the subsequent subsections.

\subsection{Continuous Interaction}

In the continuous version, the user is able to interact at anytime. With it, we aim at providing interpretational freedom to the users and to enhance their perception of the story by inspiring their curiosity and encouraging their spirit 
of exploration. Therefore, the graphical user interface for the user's typed text input is always enabled.

This version of our interactive demonstrator is a lot more complex, since agent dialogs need to be interruptive in a manner that characters are able to react to user interaction at any time. Therefore a much lager set of interaction possibilities needs to be provided, as users might interact differently during an ongoing dialog than they might interact after being asked a specific question. By providing a continuous interaction style, user interaction should be perceived a lot more natural and intuitive, since dialogs in real life work in a similar manner, too.

An important issue arising due to this interaction style is the timing of user input and system output. The dialog management tool of our system [6] provides concepts for hierarchy, concurrency, variable scoping, multiple interaction policies and a runtime history, which is needed for this kind of interaction. In the continuous version, the current dialog needs to be interruptive as a prompt reaction to the user's input is required. In case of a user input, the dialog that was currently active is stopped at utterance level and the dialog is not further executed.

For prompt reactions, the process modeling of the target of an interaction needs to be properly synchronized. After a user's utterance has been detected, the conversation either continues with another dialog or with reentering the previous dialog. For the latter, a runtime history provides the possibility to remember the last substates of the conversational flow.

With this version of the demonstrator, we provide a highly interactive system that might help engage the user. Potential issues with this version might be a confusion of the user, in case he or she does not know how or at what point in time to interact with the system-controlled characters. In addition, the impact of the user's interaction to the flow of the story might not always be clear, since virtual characters do sometimes need to block or redirect the user in order to continue with the story line.

\subsection{System-Initiated Interaction}

For the system-initiated interaction style, the story flow and dialog scripts from the continuous version were reused. However, not all states are likely to be reached, since the graphical user interface for the user's typed text input is disabled during agent conversations. Only at points in time when the user can state an opinion or advise a character, and thus make a contribution to the narrative flow, the user is actually able to communicate with the system. Thus, clear interaction prompts are provided by the characters, and only user interactions that are typical of the particular branch are likely to occur. In that manner, on the one hand the user knows what kind of input is required. On the other hand, the parsing of these sentences is easier, since the domain is limited. If the user is, for example, explicitly asked for his or her opinion on the waitress, it is likely that the user states an opinion and does not try to comment on other things. Hence, only a few of the possible characters' responses are therefore needed. 
Dialogs that are held by system-controlled characters are not interruptive in this version. Only if the end of a dialog is reached, a contribution from the user's side is possible. However, the runtime history is still maintained, since a dialog might have to be reentered, e.g. if the user walks away and approaches the target group again afterwards.

This interaction style is a lot simpler to model than the continuous version. As a possible advantage, it might be more intuitive to use for unexperienced users, since clear questions are asked at certain points in time only. Since interaction is only possible at story branches, the impact of the user's interaction on the flow of the story should be comprehensible to the user. However, this version might be perceived as boring by the users.

\section{Exploratory User Study}

An experimental user study was conducted to explore the impact of the decision for continuous or round-based user dialog on the end-user experience. Conceptually, the term "user experience" was grounded on previous theoretical and empirical work (see [1] and [2]). The framework includes diverse modes of experience such as suspense, curiosity, flow, and effectance (perceived causal influence onto the story). For this conceptual framework, a self-report measurement tool [2] was used in the present study. It was expanded by the experiential aspect of autonomy [4], which is particularly relevant to the comparison of continuous versus round-based dialog: The former might cause stronger perceptions of autonomy, whereas the latter may make users perceive constrained autonomy, as users need to 'wait' until it is their 'turn' to speak during interaction with the story characters.

A total of 42 university students (mean age: 22 years, 30 females) participated in the study. They were randomly assigned to either experience the Virtual Beergarden scenario with continuous $(n=20)$ or round-based dialog $(n=22)$. After receiving a brief introduction of the system, their exposure typically lasted for five to ten minutes. Afterwards, participants filled in a questionnaire about the various dimensions of user experience. Subsequently, they took part in another study that is not reported here, were thanked and dismissed afterwards. They received a financial compensation of overall 15 EUR for both studies.

For all scales of the user experiences, the rating questions on whether expectations were met, and the items about which conventional media experience was perceived to be similar, mean comparison of index or item means between participants exposed to either the continuous or the round-based dialog were computed. T-test statistics were applied to determine group differences of particular importance. Table 1 summarizes selected findings. Users who interacted with the system through continuous dialog reported greater perceived autonomy and curiosity, were less disappointed in terms of engagement, and rated the experience more similar to improvisation theater than the user group confronted with round-based dialog (see table 1). 
Table 1. Selected findings from exploratory user study. All items and scales used five-point ratings ranging from 1 (do not agree at all) to 5 (fully agree)

\begin{tabular}{|c|c|c|c|c|c|}
\hline \multirow[b]{2}{*}{ Dimension of user experience } & \multicolumn{2}{|c|}{$\begin{array}{c}\text { Users confronted } \\
\text { with continuous } \\
\text { dialog }\end{array}$} & \multicolumn{2}{|c|}{$\begin{array}{c}\text { Users confronted } \\
\text { with round-based } \\
\text { dialog }\end{array}$} & \multirow{2}{*}{$\begin{array}{c}\text { t-test } \\
p\end{array}$} \\
\hline & M & $\mathrm{SD}$ & $\mathrm{M}$ & $\mathrm{SD}$ & \\
\hline Autonomy & 2.68 & 0.93 & 2.17 & 0.79 & 0.055 \\
\hline Curiosity & 3.78 & 0.77 & 3.33 & 0.92 & 0.095 \\
\hline Suspense & 3.16 & 0.74 & 2.82 & 0.79 & n.s \\
\hline Enjoyment & 3.45 & 0.95 & 3.07 & 0.94 & n.s \\
\hline Comparisons with expectations & & & & & \\
\hline $\begin{array}{l}\text { "I had the expectation that the } \\
\text { experience would be more engaging" }\end{array}$ & 1.75 & 0.97 & 2.18 & 0.59 & 0.09 \\
\hline Similarities to other experiences & & & & & \\
\hline $\begin{array}{c}\text { "The experience reminded me of } \\
\text { playing a video game" }\end{array}$ & 4.25 & 1.33 & 4.82 & 0.39 & 0.06 \\
\hline $\begin{array}{l}\text { "The experience reminded me of } \\
\text { watching a movie" }\end{array}$ & 2.45 & 0.99 & 1.77 & 1.15 & 0.049 \\
\hline $\begin{array}{l}\text { "The experience reminded me a } \\
\text { little of playing improvisation theater" }\end{array}$ & 2.10 & 1.33 & 1.36 & 0.49 & 0.02 \\
\hline
\end{tabular}

\section{Conclusions}

Findings indicate that shifting between technologically quite different options does not affect user experiences in a fundamental way: Many conceptually relevant dimensions of the user experience were found to be equal in both experimental groups, and only a few (nearly) significant differences emerged. These differences are highly interesting, however: They suggest that users value continuous dialog, which comes with greater technological requirements, higher in terms of autonomy and curiosity about how the story will evolve. Moreover, users' comparisons of the interactive story with previous media experiences shift if the dialog mode is changed: Continuous dialog is perceived to be closer to film and improvisation theater experiences, whereas users judge round-based dialog to be more similar to video game play, probably to classic menu-based adventure games. So overall, the technologically more ambitious design option of continuous dialog seems to contribute to a more unique, novel kind of user experience, whereas the less demanding option of round-based dialog directs users' perceptions towards well-known experiences of interactive entertainment.

Designers can now discuss whether they want to provide a higher degree of perceived autonomy and more of an improvisation theater kind of experience to users [8] or whether they strive for an experience similar to playing a video game. Of course, standardized measures do not tell designers the full story; qualitative approaches with single users are equally important. Yet the fact that theory-based, standardized measures (such as [2]) reveal interpretable and 
relevant effects of design decisions even with prototype systems, clearly indicates that quantitative-experimental approaches in early user research can make important contributions during ongoing system development.

An interesting observation is the user's perception of how the Virtual Beergarden scenario is perceived by human users. Initially, the interactive system was designed to resemble a virtual improvisational theater [9]. Our evaluation study, however, reveals that the story reminds more of playing a video game. This perception does not necessarily need to stand in the light of an improvisational theater experience, since graphics and virtual worlds are directly adopted from video gaming. Nevertheless, our findings indicate that a continuous interactive dialog style enhanced the users' perception towards an improvisational theater experience. Although our soap story in the Virtual Beergarden scenario was not directly perceived as improvisational theater, integrating more autonomy for the user can can be seen as a step in the right direction.

Acknowledgments. This work was funded by the European Commission within the 7th Framework Program under grant agreement IRIS (FP7-ICT231824).

\section{References}

1. Klimmt, C., Roth, C., Vermeulen, I., Vorderer, P., Roth, F.S.: Forecasting the experience of future entertainment technology: "Interactive Storytelling" and media enjoyment. Games and Culture: A Journal of Interactive Media (in press)

2. Vermeulen, I.E., Roth, C., Vorderer, P., Klimmt, C.: Measuring User Responses to Interactive Stories: Towards a Standardized Assessment Tool. In: Aylett, R., Lim, M.Y., Louchart, S., Petta, P., Riedl, M. (eds.) ICIDS 2010. LNCS, vol. 6432, pp. 38-43. Springer, Heidelberg (2010)

3. Mehta, M., Dow, S., Mateas, M., MacIntyre, B.: Evaluating a conversation-centered interactive drama. In: 6th International Joint Conference on Autonomous Agents and Multiagent Systems, AAMAS 2007 (2007)

4. Ryan, R.M., Rigby, C.S., Przybylski, A.: The motivational pull of video games: A self-determination theory approach. Motivation and Emotion (30), 347-363 (2006)

5. Engel, R.: Robust and Efficient Semantic Parsing of FreeWord Order Languages in Spoken Dialogue Systems. In: Interspeech 2005 (2005)

6. Mehlmann, G.U.: SceneMaker: An Interpreter for Parallel Processes Modeling the Behavior of Virtual Characters. Master's thesis, German Research Center for Artificial Intelligence (2009)

7. Augsburg University (2011), http://mm-werkstatt.informatik.uni-augsburg.de/projects/aaa

8. Tanenbaum, T.J., Tanenbaum, K.: Improvisation and Performance as Models for Interacting with Stories. In: Spierling, U., Szilas, N. (eds.) ICIDS 2008. LNCS, vol. 5334, pp. 250-263. Springer, Heidelberg (2008)

9. Endrass, B., Boegler, M., Bee, N., André, E.: What Would You Do in Their Shoes? Experiencing Different Perspectives in an Interactive Drama for Multiple Users. In: Iurgel, I.A., Zagalo, N., Petta, P. (eds.) ICIDS 2009. LNCS, vol. 5915, pp. 258-268. Springer, Heidelberg (2009) 